\section{SUSTAINABLE REGRESSION OF DISSEMINATED SOLID TUMORS MEDIATED BY IN SITU COMBINATIONAL TREATMENT WITH ADOPTIVE T CELL THERAPY AND ONCOLYTIC ADENOVIRUS DELTA-24-RGDOX}

Hong Jiang*, Dong Ho Shin, Sagar Sohoni, Teresa Nguyen, Yanhua Yi, Xuejun Fan, Joy Gumin, Sanjay Singh, Frederick Lang, Candelaria Gomez-Manzano, Juan Fueyo. MD Anderson Cancer Center, Houston, TX, USA

Background Unlike its remarkable success in treating hematological malignancies, adoptive $\mathrm{T}$ cell therapy (ACT), such as CAR $\mathrm{T}$ therapy, targeting limited tumor-associated antigens (TAAs) is far less effective in patients with heterogeneous solid tumors. Oncolytic viruses, including oncolytic adenovirus Delta-24-RGDOX from our group [1, 2], are emerging as promising immunotherapy drugs. To take advantage of instant antitumor activity of $\mathrm{T}$ cells and oncolytic adenovirus mediated in situ autovaccination against heterogeneous cancer cells, we hypothesize that intratumorally injected Delta-24-RGDOX complements with ACT to overcome antigen escape, leading to more sustainable anti-cancer effect.

Methods We used B16-OVA-C57BL/6 subcutaneous (s.c.)/s.c. melanoma model [2] to assess the systemic therapeutic effect in disseminated tumors. OVA (or gp100)-specific CD8+ T cells were injected into the first tumor, followed by three injections of Delta-24-RGDOX into the same tumor. T cells from the mice were profiled for surface markers with flow cytometry. Activity of splenocytes against tumor cells and specific TAAs was measured with ELISA. Tumor growth was monitored through measuring tumor size three times a week. The animal survival curves were plotted according to the Kaplan-Meier method.

Results TAA-specific $\mathrm{T}$ cells injected into the first s.c. tumor showed tropism for disseminated s.c. and intracranial tumors, tumor draining lymph nodes, compared to normal tissue, spleen and peripheral blood. Delta-24-RGDOX increased total $\mathrm{T}$ cell presence within the tumors, and the activity of the $\mathrm{T}$ cells against the tumor cells and targeting other antigens than the one targeted by the transferred $\mathrm{T}$ cells. Consequentially, the combination of OVA-specific T cells and Delta-24-RGDOX was more potent to inhibit the injected first tumor and untreated disseminated second tumor growth and resulted in improved survival rate than either of the agent alone $(\mathrm{p}<$ 0.05). Interestingly, we observed relapse of the regressed tumors in the group treated by $\mathrm{T}$ cells alone. But the relapse was not observed in most of the mice in the combination group. The survivors from the combination therapy were protected from rechallenging with B16-OVA cells but not lung carcinoma cells, suggesting the development of immune memory.

Conclusions Delta-24-RGDOX collaborates with ACT to induce more potent systemic immunity against the tumors, leading to sustainable tumor regression and improved survival rate. Our study indicates the virus induces antigen spread, resulting in expansion of $\mathrm{T}$ cell repertoire to prevent cancer relapse in ACT.

\section{REFERENCES}

1. Jiang, H., et al., Localized Treatment with Oncolytic Adenovirus Delta-24-RGDOX Induces Systemic Immunity against Disseminated Subcutaneous and Intracranial Melanomas. Clin Cancer Res, 2019. 25(22): p. 6801-6814. https://cancerres.aacrjournals.org/content/77/14/3894.long.

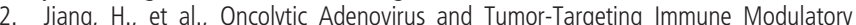
Therapy Improve Autologous Cancer Vaccination. Cancer Res, 2017. 77(14): p. 3894-3907.https://dincancerres.aacrjournals.org/content/25/22/6801.full-text.pdf 